\title{
Antimicrobial Resistance in Invasive Bacterial Infections in Hospitalized Children, Cambodia, 2007-2016
}

\author{
Andrew Fox-Lewis, Junko Takata, Thyl Miliya, Yoel Lubell, Sona Soeng, Poda Sar, Kolthida Rith, \\ Gregor McKellar, Vanaporn Wuthiekanun, Erin McGonagle, Nicole Stoesser, Catrin E. Moore, \\ Christopher M. Parry, Claudia Turner, Nicholas P.J. Day, Ben S. Cooper, Paul Turner
}

\begin{abstract}
To determine trends, mortality rates, and costs of antimicrobial resistance in invasive bacterial infections in hospitalized children, we analyzed data from Angkor Hospital for Children, Siem Reap, Cambodia, for 2007-2016. A total of 39,050 cultures yielded 1,341 target pathogens. Resistance rates were high; $82 \%$ each of Escherichia coli and Klebsiella pneumoniae isolates were multidrug resistant. Hospital-acquired isolates were more often resistant than community-acquired isolates; resistance trends over time were heterogeneous. K. pneumoniae isolates from neonates were more likely than those from nonneonates to be resistant to ampicillin-gentamicin and third-generation cephalosporins. In patients with community-acquired gram-negative bacteremia, third-generation cephalosporin resistance was associated with increased mortality rates, increased intensive care unit admissions, and 2.26-fold increased healthcare costs among survivors. High antimicrobial resistance in this setting is a threat to human life and the economy. In similar low-resource settings, our methods could be reproduced as a robust surveillance model for antimicrobial resistance.
\end{abstract}

$\mathrm{W}$ orldwide, invasive bacterial infections are a leading cause of childhood deaths, mostly in lowand middle-income countries (1). Management of such

Author affiliations: University of Oxford, Oxford, UK (A. Fox-Lewis,

J. Takata, Y. Lubell, N. Stoesser, C.E. Moore, C. Turner,

N.P.J. Day, B.S. Cooper, P. Turner); Angkor Hospital for Children,

Siem Reap, Cambodia (A. Fox-Lewis, T. Miliya, S. Soeng, P. Sar,

K. Rith, G. McKellar, C. Turner, P. Turner); Cambodia-Oxford

Medical Research Unit, Siem Reap (A. Fox-Lewis, T. Miliya,

S. Soeng, P. Sar, K. Rith, C. Turner, P. Turner); Mahidol-Oxford

Tropical Medicine Research Unit, Bangkok, Thailand (Y. Lubell,

V. Wuthiekanun, C.E. Moore, N.P.J. Day, B.S. Cooper); University

of Colorado, Aurora, Colorado, USA (E. McGonagle); Liverpool

School of Tropical Medicine, Liverpool, UK (C.M. Parry); Nagasaki

University, Nagasaki, Japan (C.M. Parry)

DOI: https://doi.org/10.3201/eid2405.171830 infections is threatened by the rising prevalence of antimicrobial resistance (AMR), particularly among neonates (2). However, data on AMR in invasive bacterial infections in children from low- and middle-income countries are scarce (3-6).

To combat the global threat of AMR, improved surveillance to detect emerging and long-term resistance trends is vital (7). Several global initiatives, such as the Fleming Fund, have been recently established to improve laboratory capacity in low- and middle-income countries $(7,8)$, and the World Health Organization (WHO) Global Antimicrobial Resistance Surveillance System (GLASS) (9) has targeted 6 invasive pathogens for routine antimicrobial resistance surveillance: Escherichia coli, Klebsiella pneumoniae, Acinetobacter baumannii, Salmonella spp., Staphylococcus aureus, and Streptococcus pneumoniae. Monitoring resistance in these pathogens is particularly important for invasive bacterial infections in children in low- and middle-income countries, where most treatment is empirically prescribed and must be based on reliable contemporaneous resistance data to be effective.

Recent systematic reviews of AMR in invasive bacterial infections in children highlight the paucity of data available and do not report temporal resistance trends $(5,6)$. In addition, although recent studies indicate excess deaths caused by AMR in low- and middle-income countries (10), there is limited evidence describing the economic and mortality burden of resistance at the patient level, particularly among children.

We analyzed 10 years of continuous AMR surveillance data for invasive bacterial infections in children from a sentinel surveillance site in Cambodia and describe resistance trends over time, by age group, and by site of acquisition (community or hospital). To evaluate the excess deaths and cost burden associated with third-generation cephalosporin resistance in community-acquired gramnegative bacteremia in hospitalized children, we analyzed patient-level data. 


\section{Methods}

\section{Study Design and Sample Selection}

Angkor Hospital for Children is an $\approx 100$-bed nongovernmental hospital in Siem Reap, Cambodia. Of children admitted, around two thirds reside in Siem Reap Province (11), where the incidence of poverty exceeds $50 \%$ (12); $93 \%$ are admitted from the community and $7 \%$ are transferred from another hospital (P. Turner, unpub data). Because this hospital has no maternity/obstetric ward, all children are born outside the hospital. Blood cultures are routinely taken from febrile (axillary temperature $>37.5^{\circ} \mathrm{C}$ ) hospitalized patients, according to clinical algorithms, at no patient cost.

We reviewed hospital microbiology data for 20072016 and extracted AMR data for selected blood culture and cerebrospinal fluid (CSF) culture isolates. Target organisms consisted of the 6 GLASS blood culture priority pathogens (9), Neisseria meningitidis (a vaccine-preventable pathogen), and non-GLASS pathogens for which $\geq 30$ organisms were isolated over the study period. We included in the study the first isolate of a given organism per patient per 14-day infection episode, except for Salmonella spp., for which we included only the first isolate per patient to avoid double counting potential relapses. Clinical data were extracted from hospital patient records. The study was approved by the Angkor Hospital for Children Institutional Review Board (AHC-IRB, 0185-17) and the Oxford Tropical Research Ethics Committee (OxTREC, 508-17).

\section{Procedures and AMR Reporting}

We processed blood and CSF culture specimens as described elsewhere $(11,13)$. Antimicrobial susceptibility testing was undertaken by disk diffusion and Etest MIC, according to Clinical and Laboratory Standards Institute guidelines (14) (online Technical Appendix Methods and Table 1, https://wwwnc.cdc.gov/EID/article/24/5/17-1830Techapp1.pdf). Resistance proportions are reported as number of resistant isolates/number of isolates tested.

\section{Outcome Analyses}

We included in patient outcome analyses community-acquired monomicrobial Enterobacteriaceae (excluding Salmonellae) and A. baumannii bacteremia. These pathogens represent common causes of sepsis in children worldwide where third-generation cephalosporins would be a firstline/empiric treatment. We obtained clinical and costing data from hospital records and calculated cost per patient as admission cost plus antimicrobial costs.

\section{Statistical Analyses}

We treated isolates from specimens taken within 48 hours of admission as community-acquired infections and after 48 hours as hospital-acquired infections. However, Salmonella enterica serotypes Typhi and Paratyphi and Burkholderia pseudomallei isolates were always considered community-acquired infections. To ensure sufficient data per period, we grouped isolates into 2-year blocks. We assessed associations between resistance and year of isolation, patient age group, and site of acquisition (community vs. hospital) by univariable and multivariable logistic regression. Multivariable models included all variables. According to assessment of model fit by calculation of Akaike information criterion and plotting of observed versus predicted data, we considered time (year of isolation) a factor unless otherwise stated.

For the outcome analyses, we used univariable analysis to compare variables by third-generation cephalosporin resistance status and patient outcome by using the Mann-Whitney-Wilcoxon rank-sum test for continuous variables and the $\chi^{2}$ test with Yates correction for categorical variables. For multivariable logistic regression, outcome variables were hospital deaths and intensive care unit (ICU) admissions, and covariates were resistance, age group, age $<10$ years, malnutrition, sex, and organism type (Enterobacteriaceae vs. A. baumannii). We conducted multivariable linear regression by using admission duration and cost for survivors as outcome variables and using the same covariates. The linear model variables were log transformed, and results are presented with log- and back-transformed coefficients, which is interpreted as a multiplicative rather than an additive model. Analyses were undertaken by using the R statistical package (15).

\section{Results}

During the 10-year study period, 39,050 sterile site samples were collected for culture: 36,358 (93.1\%) blood and 2,692 (6.9\%) CSF (online Technical Appendix Figure 1). The sampling rate, indicated by the blood culture:hospital admission ratio, rose throughout the study period as utility of the clinical microbiology service increased. Approximately 1 blood culture was sent for every 3 admissions in 2007 (1,293 blood cultures:3,829 admissions), rising to 1 blood culture per admission in 2013 (5,294 blood cultures:5,208 admissions) and subsequently remaining stable (online Technical Appendix Figure 2). From 2012 through 2016, the proportion of blood cultures from neonates rose from $9.1 \%$ to $21.2 \%$, and the proportion from children $\geq 5$ years of age dropped from $35.4 \%$ to $22.4 \%$ (online Technical Appendix Table 2).

Of the 39,050 specimens collected, 3,666 (9.4\%) were culture positive, yielding 4,028 isolates. Skin organism contamination was identified in 1,937 (5.3\%) blood cultures. Clearly pathogenic bacteria comprised $37.5 \%(1,512)$ of isolates grown, 9.1\% (366) were of uncertain significance, 
and $53.4 \%(2,150)$ were designated skin contaminants. A total of 1,341 target organisms met inclusion criteria; 1,088 (81.1\%) were GLASS pathogens and 253 (18.9\%) were non-GLASS pathogens. GLASS pathogens were Salmonella spp. (408, 30.4\%); S. aureus $(186,13.9 \%)$; S. pneumoniae (166, 12.4\%); K. pneumoniae (146, 10.9\%); E. coli (107, 8.0\%); and A. baumannii (75, 5.6\%).

\section{Overall AMR Rates}

Overall AMR rates were high, especially among gramnegative GLASS organisms (Table 1). Ampicillin-gentamicin resistance (resistance to both agents) was detected in $62.1 \%(90 / 145)$ of $K$. pneumoniae isolates and $47.2 \%$ (50/106) of E. coli isolates. Third-generation cephalosporin resistance was detected in $78.8 \%(115 / 146)$ of
$K$. pneumoniae isolates, $49.5 \%(53 / 107)$ of $E$. coli isolates, and $93.3 \%(70 / 75)$ of $A$. baumannii isolates; multidrug resistance in these 3 organisms was $81.8 \%(108 / 132)$, $82.1 \%(69 / 84)$, and $93.3 \%$ (70/75), respectively. Carbapenem resistance was uncommon: $<1 \%$ of $K$. pneumoniae $(1 / 142)$ and $E$. coli isolates $(0 / 98)$ and $13.5 \%(10 / 74)$ of A. baumannii isolates were resistant.

Resistance differed greatly among the 3 groups of Salmonella spp. The proportion of resistant isolates was highest for Salmonella Typhi: 95.7\% (308/322) were fluoroquinolone resistant, $86.0 \%$ (270/314) multidrug resistant, and $85.0 \%$ (266/313) fluoroquinolone and multidrug resistant. The least resistant group was Salmonella Paratyphi A: $22.7 \%$ (10/44) of isolates were fluoroquinolone resistant and none were multidrug resistant $(0 / 43)$.

\begin{tabular}{|c|c|c|c|c|c|c|}
\hline \multirow[b]{2}{*}{ Pathogen, resistance type } & \multirow{2}{*}{$\begin{array}{c}\text { No. isolates } \\
\text { resistant/no. tested (\%) }\end{array}$} & \multicolumn{5}{|c|}{ Year of isolation } \\
\hline & & $2007-2008$ & $2009-2010$ & $2011-2012$ & 2013-2014 & $2015-2016$ \\
\hline \multicolumn{7}{|l|}{ Gram-negative } \\
\hline Klebsiella pneumoniae & 146 & 11 & 17 & 56 & 42 & 20 \\
\hline AMP-GEN† & $90 / 145(62.1)$ & $5 / 11(45.5)$ & $10 / 16(62.5)$ & $46 / 56(82.1)$ & $26 / 42(61.9)$ & $3 / 20(15.0)$ \\
\hline $3 G C$ & $115 / 146(78.8)$ & 8/11 (72.7) & $13 / 17(76.5)$ & $50 / 56(89.3)$ & $37 / 42(88.1)$ & $7 / 20(35.0)$ \\
\hline Carbapenem & $1 / 142(0.7)$ & $0 / 8$ & $0 / 16$ & $0 / 56$ & $1 / 42(2.4)$ & $0 / 20$ \\
\hline Multidrug & $108 / 132(81.8)$ & $8 / 8(100)$ & $12 / 12(100)$ & $45 / 50(90.0)$ & $36 / 42(85.7)$ & $7 / 20(35.0)$ \\
\hline Escherichia coli & 107 & 12 & 22 & 21 & 30 & 22 \\
\hline AMP-GEN & $50 / 106(47.2)$ & $4 / 12(33.3)$ & $12 / 21(57.1)$ & $8 / 21(38.1)$ & $17 / 30(56.7)$ & $9 / 22(40.9)$ \\
\hline AMP & $101 / 107(94.4)$ & $10 / 12(83.3)$ & $21 / 22(95.5)$ & $21 / 21(100)$ & $28 / 30(93.3)$ & $21 / 22(95.5)$ \\
\hline GEN & $51 / 106(48.1)$ & $4 / 12(33.3)$ & $12 / 21(57.1)$ & $8 / 21(38.1)$ & $18 / 30(60.0)$ & $9 / 22(40.9)$ \\
\hline $3 G C$ & $53 / 107(49.5)$ & $3 / 12(25.0)$ & $11 / 22(50.0)$ & $11 / 21(52.4)$ & $16 / 30(53.3)$ & $12 / 22(54.5)$ \\
\hline Carbapenem & $0 / 98$ & $0 / 3$ & $0 / 22$ & $0 / 21$ & $0 / 30$ & $0 / 22$ \\
\hline Multidrug & $69 / 84(82.1)$ & $3 / 3(100)$ & $13 / 13(100)$ & $15 / 16(93.8)$ & 23/30 (76.7) & $15 / 22(68.2)$ \\
\hline Acinetobacter baumannii & 75 & 2 & 7 & 30 & 27 & 9 \\
\hline $3 G C$ & 70/75 (93.3) & $2 / 2(100)$ & 6/7 (85.7) & 27/30 (90.0) & $27 / 27(100)$ & 8/9 (88.9) \\
\hline Carbapenem & $10 / 74(13.5)$ & $1 / 2(50.0)$ & $1 / 6(16.7)$ & $5 / 30(16.7)$ & $3 / 27(11.1)$ & $0 / 9$ \\
\hline Multidrug & $21 / 71$ (29.6) & $1 / 2(50.0)$ & $2 / 6(33.3)$ & $9 / 27(33.3)$ & $8 / 27(29.6)$ & $1 / 9(11.1)$ \\
\hline Salmonella Typhi & 323 & 44 & 51 & 146 & 40 & 42 \\
\hline $\mathrm{FQ}$ & 308/322 (95.7) & $39 / 44$ (88.6) & $48 / 51(94.1)$ & $139 / 145(95.9)$ & $40 / 40(100)$ & $42 / 42(100)$ \\
\hline CRO & $1 / 173(0.6)$ & $0 / 44$ & $1 / 21(4.8)$ & $0 / 26$ & $0 / 40$ & $0 / 42$ \\
\hline MDR & $270 / 314(86.0)$ & $31 / 41(75.6)$ & $39 / 47$ (83.0) & $134 / 144(93.1)$ & $35 / 40(87.5)$ & $31 / 42(73.8)$ \\
\hline FQ and multidrug & $266 / 313(85.0)$ & $30 / 41(73.2)$ & $38 / 47$ (80.9) & $132 / 143(92.3)$ & $35 / 40(87.5)$ & $31 / 42(73.8)$ \\
\hline Salmonella Paratyphi A & 44 & 3 & 0 & 0 & 35 & 6 \\
\hline $\mathrm{FQ}$ & $10 / 44(22.7)$ & $3 / 3(100)$ & & & 4/35 (11.4) & $3 / 6(50.0)$ \\
\hline CRO & $0 / 44$ & $0 / 3$ & & & $0 / 35$ & $0 / 6$ \\
\hline MDR & $0 / 43$ & $0 / 2$ & & & $0 / 35$ & $0 / 6$ \\
\hline FQ and multidrug & $0 / 0$ & $0 / 0$ & & & $0 / 0$ & $0 / 0$ \\
\hline Non-Typhoid Salmonellae & 41 & 7 & 4 & 7 & 9 & 14 \\
\hline$F Q$ & 26/41 (63.4) & 4/7 (57.1) & $2 / 4(50.0)$ & 4/7 (57.1) & 6/9 (66.7) & $10 / 14(71.4)$ \\
\hline CRO & $3 / 37(8.1)$ & $0 / 7$ & $0 / 4$ & $1 / 3(33.3)$ & $0 / 9$ & 2/14 (14.3) \\
\hline Multidrug & 9/39 (23.1) & $3 / 7$ (42.9) & $1 / 2(50.0)$ & $2 / 7(28.6)$ & 2/9 (22.2) & $1 / 14(7.1)$ \\
\hline FQ and multidrug & $5 / 39(12.8)$ & 3/7 (42.9) & $1 / 2(50.0)$ & $0 / 7$ & $0 / 9$ & $1 / 14(7.1)$ \\
\hline \multicolumn{7}{|l|}{ Gram-positive } \\
\hline Staphylococcus aureus & 186 & 26 & 38 & 43 & 42 & 37 \\
\hline MET & 24/185 (13.0) & $3 / 26(11.5)$ & $4 / 38(10.5)$ & $8 / 42(19.0)$ & $3 / 42(7.1)$ & $6 / 37(16.2)$ \\
\hline VAN & $0 / 9$ & $0 / 0$ & $0 / 0$ & $0 / 0$ & $0 / 3$ & $0 / 6$ \\
\hline Streptococcus pneumoniae & 166 & 17 & 36 & 40 & 41 & 32 \\
\hline Penicillin & $73 / 144(50.7)$ & $5 / 9(55.6)$ & $10 / 23(43.5)$ & 16/39 (41.0) & $20 / 41(48.8)$ & $22 / 32(68.8)$ \\
\hline MAC/LIN & 49/165 (29.7) & $5 / 17(29.4)$ & $10 / 35(28.6)$ & $12 / 40(30.0)$ & $11 / 41(26.8)$ & $11 / 32(34.4)$ \\
\hline MDR & $63 / 93(67.7)$ & $0 / 0$ & $0 / 0$ & $10 / 20(50.0)$ & $26 / 41(63.4)$ & $27 / 32(84.4)$ \\
\hline
\end{tabular}


Resistance in nontyphoidal Salmonella spp. fell between that of Salmonella Typhi and Paratyphi A: 63.4\% (26/41) of isolates were fluoroquinolone resistant, $23.1 \%(9 / 39)$ multidrug resistant, and $12.8 \%(5 / 39)$ fluoroquinolone and multidrug resistant. Only 1.6\% (4/254) of Salmonella spp. isolates were ceftriaxone resistant.

In gram-positive GLASS organisms, approximately one third of $S$. pneumoniae isolates were macrolide/lincosamide resistant $(29.7 \%, 49 / 165)$, half were penicillin resistant $(50.7 \%, 73 / 144)$, and two thirds were multidrug resistant $(67.8 \%, 63 / 93)$. Only $13.0 \%(24 / 185)$ of $S$. aureus isolates were methicillin resistant.

The most frequently isolated non-GLASS pathogen was Burkholderia pseudomallei, the causative agent of melioidosis $(26.1 \%, 66)$, which was universally sensitive to the first-line drugs ceftazidime and co-trimoxazole (Table 2). Next was Haemophilus influenzae, for which approximately half of isolates were ampicillin resistant $(53.6 \%, 30 / 56)$ and one third multidrug resistant $(37.1 \%$, 13/35), followed by Enterobacter cloacae, which had a similar resistance profile to $K$. pneumoniae and E. coli. The remaining non-GLASS pathogens (group A Streptococcus, Pseudomonas aeruginosa, and Neisseria meningitides) exhibited low-level resistance to the key antimicrobials reported.

\section{AMR Time Trends}

The most frequently isolated organisms were $K$. pneumoniae, E. coli, Salmonella Typhi, S. aureus, and $S$. pneumoniae; AMR time trends were heterogeneous (Tables 1, 3-5; Figure 1). S. pneumoniae penicillin resistance fluctuated over time; $55.6 \%$ (5/9) of isolates were resistant in 2007-2008, dropping to $41.0 \%(16 / 39)$ in 2011-2012 before rising to $68.8 \%(22 / 32)$ in $2015-2016$. During 2011-2016, when we tested S. pneumoniae for multidrug resistance, the proportion of multidrug-resistant $S$. pneumoniae isolates increased from $50.0 \%$ to $84.4 \%$. Salmonella Typhi fluoroquinolone resistance also increased over the study period, from $88.6 \%$ to $100 \%$. Multivariable logistic regression analysis in which time was a continuous variable showed an increased probability of Salmonella Typhi fluoroquinolone resistance over time (adjusted odds ratio [aOR] 2.14, 95\% CI 1.29-3.74; $p=0.005$ ), although not statistically significant in the model when time was a factor (online Technical Appendix Table 3 and Figure 3). Conversely, during 2015-2016, the proportion of resistant $K$. pneumoniae isolates fell dramatically for most antimicrobials tested, a phenomenon not seen for $E$. coli (online Technical Appendix Table 4). For E. coli, ampicillin-gentamicin and third-generation cephalosporin resistance remained stable at $\approx 50 \%$ with no evidence of significant change over time, as did rates of methicillinresistant $S$. aureus, which remained low throughout the study period. The proportion of $K$. pneumoniae isolates from neonates peaked in $2011-2014$ at $46 \%-50 \%$ before dropping in 2015-2016 to 35\% (online Technical Appendix Table 5), paralleling the change in the proportion of resistant isolates seen. To determine any subtle shifts in susceptibility, we examined changes in zone diameter distribution over time for E. coli and K. pneumoniae and found no clear trends (online Technical Appendix Table 6, Figures 4, 5).

Table 2. Resistance proportions by year of isolation for the 253 non-Global Antimicrobial Resistance Surveillance System pathogens isolated from children at Angkor Hospital for Children, Siem Reap, Cambodia, 2007-2016*

\begin{tabular}{|c|c|c|c|c|c|c|}
\hline \multirow[b]{2}{*}{ Pathogen, resistance type } & \multirow{2}{*}{$\begin{array}{c}\text { No. isolates } \\
\text { resistant/no. tested (\%) }\end{array}$} & \multicolumn{5}{|c|}{ Year of isolation } \\
\hline & & $2007-2008$ & 2009-2010 & 2011-2012 & 2013-2014 & $2015-2016$ \\
\hline Burkholderia pseudomallei & 66 & 6 & 10 & 13 & 22 & 15 \\
\hline CAZ & $0 / 66$ & $0 / 6$ & $0 / 10$ & $0 / 13$ & $0 / 22$ & $0 / 15$ \\
\hline TMP/SXT & $0 / 61$ & $0 / 2$ & $0 / 10$ & $0 / 12$ & $0 / 22$ & $0 / 15$ \\
\hline Haemophilus influenzae & 57 & 15 & 15 & 9 & 12 & 6 \\
\hline AMP & $30 / 56(53.6)$ & $5 / 14(35.7)$ & $10 / 15(66.7)$ & $7 / 9(77.8)$ & $8 / 12(66.7)$ & $0 / 6$ \\
\hline CRO & $3 / 57(5.3)$ & $1 / 15(6.7)$ & $1 / 15(6.7)$ & $0 / 9$ & $1 / 12(8.3)$ & $0 / 6$ \\
\hline Multidrug & $13 / 35(37.1)$ & $0 / 0$ & $5 / 10(50.0)$ & $5 / 7(71.4)$ & $3 / 12(25.0)$ & $0 / 6$ \\
\hline Enterobacter cloacae & 42 & 2 & 6 & 8 & 17 & 9 \\
\hline AMP-GEN & $19 / 42(45.2)$ & $1 / 2(50.0)$ & $5 / 6(83.3)$ & $5 / 8(62.5)$ & $6 / 17$ (35.3) & $2 / 9(22.2)$ \\
\hline $3 G C$ & $34 / 42(81.0)$ & $1 / 2(50.0)$ & $5 / 6(83.3)$ & $7 / 8(87.5)$ & $14 / 17(82.4)$ & $7 / 9(77.8)$ \\
\hline Carbapenem & $3 / 41(7.3)$ & $0 / 1$ & $0 / 6$ & $0 / 8$ & $2 / 17(11.8)$ & $1 / 9(11.1)$ \\
\hline Multidrug & $18 / 37(48.6)$ & $1 / 1(100)$ & $2 / 2(100)$ & $5 / 8(62.5)$ & $7 / 17(41.2)$ & $3 / 9(33.3)$ \\
\hline Group A Streptococcus & 38 & 2 & 6 & 6 & 13 & 11 \\
\hline MAC/LIN & $6 / 37(16.2)$ & $0 / 2$ & $1 / 5(20.0)$ & $0 / 6$ & $2 / 13(15.4)$ & $3 / 11(27.3)$ \\
\hline Pseudomonas aeruginosa & 37 & 7 & 6 & 7 & 9 & 8 \\
\hline CAZ & $4 / 34(11.8)$ & $0 / 4$ & $1 / 6(16.7)$ & $1 / 7(14.3)$ & $2 / 9(22.2)$ & $0 / 8$ \\
\hline Carbapenem & $2 / 30(6.7)$ & $0 / 1$ & $0 / 5$ & $1 / 7(14.3)$ & $0 / 9$ & $1 / 8(12.5)$ \\
\hline Multidrug & $0 / 29$ & $0 / 0$ & $0 / 5$ & $0 / 7$ & $0 / 9$ & $0 / 8$ \\
\hline Neisseria meningitidis & 13 & 6 & 3 & 0 & 2 & 2 \\
\hline CRO & $1 / 13(7.7)$ & $0 / 6$ & $1 / 3(33.3)$ & & $0 / 2$ & $0 / 2$ \\
\hline
\end{tabular}

${ }^{*}$ Resistance proportions have been reported as number of resistant isolates out of number of isolates tested. Blank cell indicates that no organisms were tested during that period and, thus, the proportion of resistant organisms is unknown. 3GC, third-generation cephalosporin; AMP-GEN, resistance to both ampicillin and gentamicin; CAZ, ceftazidime; CRO, ceftriaxone; MAC/LIN, resistance to macrolides and/or lincosamides; TMP/SXT, trimethoprim/sulfamethoxazole. 


\section{AMR by Patient Age Group}

Isolates from younger children were more often resistant to clinically important antimicrobials (Tables 3-5; Figure 2; online Technical Appendix Tables 7-9). Multivariable logistic regression controlling for year of isolation and site of acquisition indicated that $K$. pneumoniae isolates from neonates were $>7$ times more likely to be resistant to the first-line treatment agents ampicillin-gentamicin

Table 3. Logistic regression analysis of resistance trends for the gram-negative Global Antimicrobial Resistance Surveillance System pathogens Klebsiella pneumoniae and Escherichia coli isolated from children at Angkor Hospital for Children, Siem Reap, Cambodia, 2007-2016*

\begin{tabular}{|c|c|c|c|c|}
\hline \multirow{2}{*}{$\begin{array}{l}\text { Pathogen, resistance type, } \\
\text { predictor variable }\end{array}$} & \multicolumn{2}{|c|}{ Univariable analysis } & \multicolumn{2}{|c|}{ Multivariable analysis } \\
\hline & OR $(95 \% \mathrm{Cl})$ & $p$ value & OR $(95 \% \mathrm{Cl})$ & $\mathrm{p}$ value \\
\hline \multicolumn{5}{|l|}{ Klebsiella pneumoniae } \\
\hline \multicolumn{5}{|l|}{ AMP-GEN } \\
\hline \multicolumn{5}{|l|}{ Year of isolation } \\
\hline $2007-2008$ & Ref & Ref & Ref & Ref \\
\hline 2009-2010 & $2.00(0.42-10.03)$ & 0.384 & $1.31(0.23-7.88)$ & 0.765 \\
\hline 2011-2012 & $5.52(1.41-22.90)$ & 0.015 & $2.61(0.58-12.45)$ & 0.213 \\
\hline 2013-2014 & $1.95(0.51-7.80)$ & 0.329 & $0.59(0.12-2.85)$ & 0.504 \\
\hline 2015-2016 & $0.21(0.03-1.12)$ & 0.075 & $0.06(0.01-0.41)$ & 0.006 \\
\hline \multicolumn{5}{|l|}{ Patient age } \\
\hline Nonneonate & Ref & Ref & Ref & Ref \\
\hline Neonate $†$ & $5.63(2.61-13.10)$ & $<0.001$ & $7.30(2.75-22.47)$ & $<0.001$ \\
\hline \multicolumn{5}{|l|}{ Infection typeł } \\
\hline Community-acquired & Ref & Ref & Ref & Ref \\
\hline Hospital-acquired & $3.87(1.81-8.51)$ & $<0.001$ & $3.62(1.42-9.58)$ & 0.008 \\
\hline \multicolumn{5}{|l|}{ 3GC } \\
\hline \multicolumn{5}{|l|}{ Year of isolation } \\
\hline 2007-2008 & Ref & Ref & Ref & Ref \\
\hline 2009-2010 & $1.22(0.20-7.02)$ & 0.823 & $0.87(0.13-5.60)$ & 0.881 \\
\hline 2011-2012 & $3.13(0.57-14.69$ & 0.156 & $1.37(0.23-7.16)$ & 0.716 \\
\hline 2013-2014 & $2.78(0.49-13.93)$ & 0.218 & $0.97(0.15-5.58)$ & 0.973 \\
\hline 2015-2016 & $0.20(0.03-0.94)$ & 0.052 & $0.06(0.01-0.39)$ & 0.005 \\
\hline \multicolumn{5}{|l|}{ Patient age } \\
\hline Nonneonate & Ref & Ref & Ref & Ref \\
\hline Neonate & $6.41(2.32-22.70)$ & 0.001 & $7.50(2.16-35.00)$ & 0.004 \\
\hline \multicolumn{5}{|l|}{ Infection type } \\
\hline Community-acquired & Ref & Ref & Ref & Ref \\
\hline Hospital-acquired & $4.04(1.76-9.44)$ & 0.001 & $3.51(1.27-10.12)$ & 0.017 \\
\hline \multirow{2}{*}{\multicolumn{5}{|c|}{$\begin{array}{l}\text { Escherichia coli } \\
\text { AMP_GEN }\end{array}$}} \\
\hline & & & & \\
\hline \multicolumn{5}{|l|}{ Year of isolation } \\
\hline 2007-2008 & Ref & Ref & Ref & Ref \\
\hline 2009-2010 & $2.67(0.63-12.75)$ & 0.194 & $2.86(0.66-14.13)$ & 0.174 \\
\hline 2011-2012 & $1.23(0.28-5.86)$ & 0.785 & $0.95(0.20-4.75)$ & 0.947 \\
\hline 2013-2014 & $2.62(0.67-11.63)$ & 0.179 & $2.25(0.53-10.72)$ & 0.282 \\
\hline 2015-2016 & $1.38(0.33-6.50)$ & 0.665 & $1.03(0.22-5.11)$ & 0.975 \\
\hline \multicolumn{5}{|l|}{ Patient age } \\
\hline Nonneonate & Ref & Ref & Ref & Ref \\
\hline Neonate & $1.04(0.42-2.57)$ & 0.924 & $0.75(0.27-2.01)$ & 0.568 \\
\hline \multicolumn{5}{|l|}{ Infection type } \\
\hline Community-acquired & Ref & Ref & Ref & Ref \\
\hline Hospital-acquired & $2.33(1.04-5.33)$ & 0.041 & $2.92(1.21-7.44)$ & 0.020 \\
\hline \multicolumn{5}{|l|}{ 3GC } \\
\hline \multicolumn{5}{|l|}{ Year of isolation } \\
\hline 2007-2008 & Ref & Ref & Ref & Ref \\
\hline 2009-2010 & $3.00(0.68-16.38)$ & 0.165 & $4.04(0.79-26.54)$ & 0.112 \\
\hline 2011-2012 & $3.30(0.74-18.21)$ & 0.134 & $2.47(0.45-16.60)$ & 0.319 \\
\hline 2013-2014 & $3.43(0.83-17.83)$ & 0.105 & $3.07(0.60-19.99)$ & 0.201 \\
\hline $2015-2016$ & $3.60(0.82-19.73)$ & 0.106 & $2.44(0.45-16.13)$ & 0.319 \\
\hline \multicolumn{5}{|l|}{ Patient age } \\
\hline Nonneonate & Ref & Ref & Ref & Ref \\
\hline Neonate & $0.92(0.37-2.27)$ & 0.861 & $0.41(0.12-1.26)$ & 0.131 \\
\hline Infection type & & & & \\
\hline Community-acquired & Ref & Ref & Ref & Ref \\
\hline Hospital-acquired & $7.50(3.09-20.01)$ & $<0.001$ & $10.14(3.70-32.14)$ & $<0.001$ \\
\hline
\end{tabular}

*3GC, third-generation cephalosporin; AMP-GEN, resistance to both ampicillin and gentamicin; OR, odds ratio; ref, referent. †Neonate, $0-28 \mathrm{~d}$ of age.

łlsolates were defined as hospital-acquired if taken $>48$ hours after admission. 
Table 4. Logistic regression analysis of resistance trends for the gram-negative Global Antimicrobial Resistance Surveillance System pathogen Salmonella enterica serovar Typhi isolated from children at Angkor Hospital for Children, Siem Reap, Cambodia, 2007-2016*

\begin{tabular}{|c|c|c|c|c|}
\hline \multirow{2}{*}{$\begin{array}{l}\text { Resistance type, predictor } \\
\text { variable }\end{array}$} & \multicolumn{2}{|c|}{ Univariable analysis } & \multicolumn{2}{|c|}{ Multivariable analysis } \\
\hline & OR $(95 \% \mathrm{Cl})$ & $p$ value & OR $(95 \% \mathrm{Cl})$ & $p$ value \\
\hline \multicolumn{5}{|l|}{ Fluoroquinolone } \\
\hline \multicolumn{5}{|l|}{ Year of isolation } \\
\hline $2007-2008$ & Ref & Ref & Ref & Ref \\
\hline 2009-2010 & $2.05(0.47-10.51)$ & 0.345 & $1.85(0.42-9.59)$ & 0.422 \\
\hline 2011-2012 & $2.97(0.82-10.37)$ & 0.085 & $3.05(0.83-10.74)$ & 0.080 \\
\hline 2013-2014 & $4.03 \times 10^{7}\left(6.26 \times 10^{45}-\infty\right)$ & 0.992 & $3.47 \times 10^{7}\left(9.49 \times 10^{-44}-\infty\right)$ & 0.992 \\
\hline 2015-2016 & $4.03 \times 10^{7}\left(1.11 \times 10^{-43}-\infty\right)$ & 0.992 & $4.14 \times 10^{7}\left(6.26 \times 10^{-43}-\infty\right)$ & 0.991 \\
\hline \multicolumn{5}{|l|}{ Patient age, y } \\
\hline$\geq 5$ & Ref & Ref & Ref & Ref \\
\hline$<5$ & $4.48(0.87-82.12)$ & 0.151 & $4.57(0.87-84.30)$ & 0.150 \\
\hline \multicolumn{5}{|l|}{ Multidrug } \\
\hline \multicolumn{5}{|l|}{ Year of isolation } \\
\hline 2007-2008 & Ref & Ref & Ref & Ref \\
\hline 2009-2010 & $1.57(0.56-4.58)$ & 0.395 & $1.45(0.50-4.29)$ & 0.491 \\
\hline 2011-2012 & 4.32 (1.64-11.44) & 0.003 & 4.55 (1.71-12.17) & 0.002 \\
\hline 2013-2014 & $2.26(0.72-7.92)$ & 0.175 & $2.08(0.65-7.41)$ & 0.228 \\
\hline 2015-2016 & $0.91(0.33-2.46)$ & 0.850 & $0.95(0.34-2.62)$ & 0.927 \\
\hline \multicolumn{5}{|l|}{ Patient age, y } \\
\hline$\geq 5$ & Ref & Ref & Ref & Ref \\
\hline$<5$ & $2.94(1.22-8.79)$ & 0.029 & $3.16(1.28-9.57)$ & 0.022 \\
\hline
\end{tabular}

(aOR 7.30, 95\% CI 2.75-22.47) and third-generation cephalosporins (aOR 7.50, 95\% CI 2.16-35.00) than were isolates from nonneonates. Similarly, S. pneumoniae isolates were more likely to be penicillin resistant (aOR 3.87, 95\% CI 1.77-8.83) and Salmonella Typhi isolates more likely to be multidrug resistant (aOR 3.16, 95\% CI 1.289.57) among children $<5$ years of age than among those $\geq 5$ years of age.

\section{AMR by Site of Infection Acquisition}

Approximately four fifths of included isolates were from community-acquired infections $(1,089,81.2 \%)$ and one fifth from hospital-acquired infections $(252,18.8 \%)$. In almost all instances, the proportion of hospital-acquired isolates resistant to a given antimicrobial was higher than that of community-acquired isolates (online Technical Appendix Tables 10-12). K. pneumoniae, the main cause of hospital-acquired infections, was $>3$ times more likely to be resistant to ampicillin-gentamicin (aOR 3.62, 95\% CI 1.42-9.58) and third-generation cephalosporins (aOR 3.51, 95\% CI 1.27-10.12) in hospital-acquired isolates (Tables $3,4)$. Increased likelihood of resistance among hospitalacquired isolates was also found for $E$. coli ampicillin-gentamicin and third-generation cephalosporin resistance and $S$. aureus methicillin resistance.

\section{Outcomes}

We analyzed patient outcomes for 129 admission episodes for community-acquired monomicrobial gram-negative bacteremia (online Technical Appendix Figure 6). Of these, $63(48.8 \%)$ isolates were resistant to third-generation cephalosporins and 34 admissions (26.4\%) resulted in patient death. Isolates consisted of E. coli $(48,37.2 \%), K$. pneumoniae $(31,24.0 \%)$, A. baumannii $(29,22.5 \%)$, and other pathogenic Enterobacteriaceae (21, 16.3\%). Neonates accounted for $26.4 \%$ (34) of the cases; median age was 8.6 months (interquartile range [IQR] 0.8-29.2 months).

Children from whom third-generation cephalosporinresistant bacteria were isolated were less likely than other patients to have received appropriate antimicrobial therapy $(57 \%$ vs. $94 \% ; p<0.001)$. If appropriate therapy was received, it was initiated later for children infected with third-generation-resistant than third-generation-sensitive organisms ( 2 days vs. 0 days after admission for those who survived $[p<0.001] ; 0.5$ days vs. 0 days for those who died $[\mathrm{p}=0.004])$. Patients who died were younger (median age 1.4 vs. 10.2 months; $\mathrm{p}=0.002$ ), were more likely to have been admitted to an ICU ( $88 \%$ vs. $27 \%$; $\mathrm{p}<0.001$ ), stayed for a shorter time in hospital ( 3 vs. 8 days; $\mathrm{p}<0.001$ ), and were more likely to have been infected with Enterobacteriaceae than A. baumannii $197 \%$ vs. $71 \% ; \mathrm{p}=0.003$ ) (online Technical Appendix Table 13). A. baumannii infections were associated with high levels of third-generation cephalosporin resistance (90\%) but a low mortality rate (3\%) despite only $48 \%$ of patients having received appropriate antimicrobials. Conversely, Enterobacteriaceae infections were associated with a high mortality rate $(33 \%)$ despite $84 \%$ of patients having received appropriate antimicrobials (online Technical Appendix Table 14).

Multivariable logistic regression (Table 6) showed that third-generation cephalosporin resistance was associated with death $(\mathrm{aOR} 2.65,95 \%$ CI $1.05-6.96 ; \mathrm{p}=0.042)$ and ICU admission (aOR 3.17, 95\% CI 1.31-8.10; $\mathrm{p}=$ 
Table 5. Logistic regression analysis of resistance trends for the gram-positive Global Antimicrobial Resistance Surveillance System pathogens Staphylococcus aureus and Streptococcus pneumoniae isolated from children at Angkor Hospital for Children, Siem Reap, Cambodia, 2007-2016*

\begin{tabular}{|c|c|c|c|c|}
\hline \multirow{2}{*}{$\begin{array}{l}\text { Pathogen, resistance type, } \\
\text { predictor variable }\end{array}$} & \multicolumn{2}{|c|}{ Univariable analysis } & \multicolumn{2}{|c|}{ Multivariable analysis } \\
\hline & OR $(95 \% \mathrm{Cl})$ & $p$ value & OR ratio $(95 \% \mathrm{Cl})$ & $p$ value \\
\hline \multicolumn{5}{|l|}{ S. aureus } \\
\hline \multicolumn{5}{|l|}{ Methicillin } \\
\hline \multicolumn{5}{|l|}{ Year of isolation } \\
\hline 2007-2008 & Ref & Ref & Ref & Ref \\
\hline 2009-2010 & $0.90(0.18-4.93)$ & 0.899 & $1.26(0.23-7.59)$ & 0.787 \\
\hline 2011-2012 & $1.80(0.47-8.90)$ & 0.418 & $2.64(0.62-14.48)$ & 0.215 \\
\hline 2013-2014 & $0.59(0.10-3.42)$ & 0.538 & $0.66(0.10-4.19)$ & 0.649 \\
\hline 2015-2016 & $1.48(0.35-7.61)$ & 0.603 & $1.84(0.39-10.47)$ & 0.455 \\
\hline \multicolumn{5}{|l|}{ Patient age $†$} \\
\hline Nonneonate & Ref & Ref & Ref & Ref \\
\hline Neonate & $0.18(0.01-0.88)$ & 0.094 & $0.14(0.01-0.75)$ & 0.064 \\
\hline \multicolumn{5}{|l|}{ Infection type } \\
\hline Community-acquired & Ref & Ref & Ref & Ref \\
\hline Hospital-acquired & $6.21(2.16-17.43)$ & $<0.001$ & $7.80(2.51-24.81)$ & $<0.001$ \\
\hline \multicolumn{5}{|l|}{ S. pneumoniae§ } \\
\hline \multicolumn{5}{|l|}{ Penicillin } \\
\hline \multicolumn{5}{|l|}{ Year of isolation } \\
\hline $2007-2008$ & Ref & Ref & Ref & Ref \\
\hline $2009-2010$ & $0.60(0.12-2.90)$ & 0.525 & $0.70(0.13-3.66)$ & 0.669 \\
\hline 2011-2012 & $0.52(0.11-2.28)$ & 0.385 & $0.42(0.08-1.95)$ & 0.269 \\
\hline 2013-2014 & $0.72(0.16-3.12)$ & 0.663 & $0.77(0.16-3.57)$ & 0.737 \\
\hline $2015-2016$ & $1.87(0.38-8.77)$ & 0.424 & $1.89(0.36-9.59)$ & 0.436 \\
\hline \multicolumn{5}{|l|}{ Patient age, y } \\
\hline$\geq 5$ & Ref & Ref & Ref & Ref \\
\hline$<5$ & $3.40(1.63-7.39)$ & 0.001 & $3.87(1.77-8.83)$ & $<0.001$ \\
\hline
\end{tabular}

${ }^{*} \mathrm{OR}$, odds ratio; ref, referent.

†Ages are grouped into neonate (0-28 d) vs. nonneonate ( $\geq 29 \mathrm{~d})$ or $<5 \mathrm{y}$ vs. $\geq 5 \mathrm{y}$, as appropriate for the organism.

flsolates were defined as hospital-acquired if taken $>48$ hours after patient admission.

$\S$ Analysis included community-acquired Streptococcus pneumoniae isolates only $(n=160)$.

0.013). Multivariable linear regression (online Technical Appendix Table 15) controlling for the same variables also showed an association between length of hospital stay among survivors and third-generation cephalosporin resistance (1.69-fold increase, 95\% CI 1.21-2.37). Third-generation cephalosporin resistance was associated with a 2.26 -fold increase in hospital costs among survivors (95\% CI 1.51-3.36) (online Technical Appendix Tables 16, 17). According to this model, the median cost per admission would have been US \$432.00 (IQR $\$ 333.30-\$ 613.90)$ if all infections were third-generation cephalosporin sensitive and US \$974.10 (IQR \$751.60$\$ 1,384.30$ ) if all infections were third-generation cephalosporin resistant.

\section{Discussion}

In this hospitalized population of children in Cambodia, AMR levels were high, particularly among the gram-negative GLASS pathogens K. pneumoniae, E. coli, and A. baumannii. These organisms exhibited concerning resistance to WHO-recommended first-line sepsis treatment, emphasizing the urgent need for revised treatment guidelines (4). Few studies inform prevalence estimates of antimicrobial resistance in low- and middle-income countries in Asia, but compared with what is known, the high levels of gramnegative resistance reported here are not uncommon $(6,16)$.
For the gram-positive GLASS pathogens, S. pneumoniae resistance was broadly similar to that of the wider region (67.7\% vs. $59.3 \%$ multidrug resistance, respectively) (17). Rates of methicillin-resistant $S$. aureus were comparatively lower; only $40.0 \%$ of hospital-acquired isolates were methicillin resistant compared with a regional average of $67.4 \%(18)$.

A major strength of this study is the observation of resistance trends over an extended period, something rarely possible in low- and middle-income countries because of lack of longstanding microbiology services. We found heterogeneous trends in resistance over time; resistance increased in some organisms (Salmonella Typhi) and decreased in others ( $K$. pneumoniae). The most surprising temporal trend observed was a drop in the proportion of resistant $K$. pneumoniae isolates for most antimicrobials tested, in contrast to largely stable resistance levels in $E$. coli. For K. pneumoniae resistance by site of acquisition, in community-acquired isolates, resistance sharply declined in 2015-2016, perhaps suggesting loss of a plasmid coding for multiple resistance determinants. Confirming this trend will require a larger dataset from multiple sites in Cambodia and further analysis of the underlying resistance mechanisms at work using a method such as whole-genome sequencing. The genetic determinants of resistance in colonizing $K$. pneumoniae and E. coli isolates from 

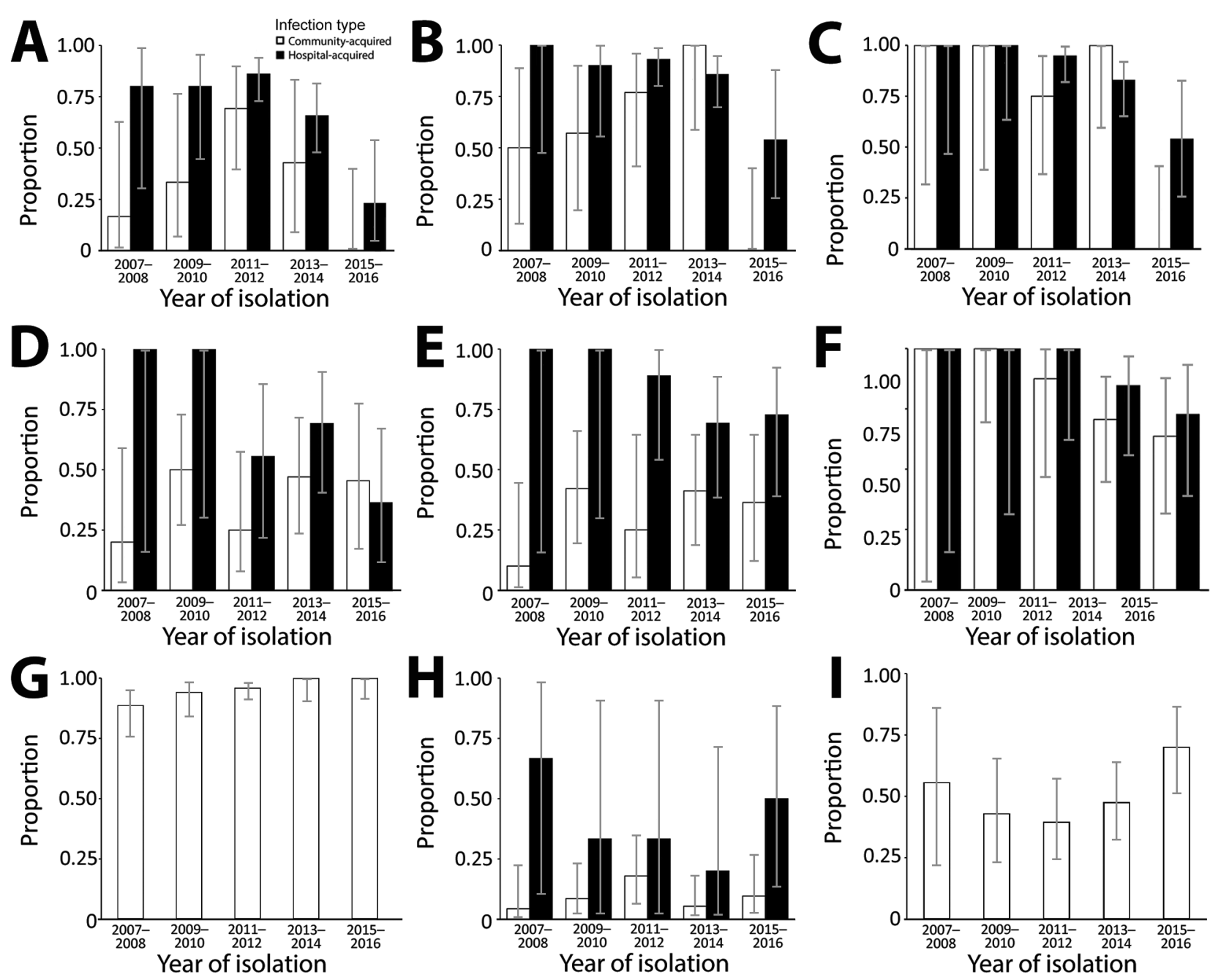

Figure 1. Antimicrobial resistance time trends, shown as proportion of resistant isolates from community-acquired and hospital-acquired infections, by year of isolation, in children at Angkor Hospital for Children, Siem Reap, Cambodia, 2007-2016. A) Klebsiella pneumoniae ampicillin-gentamicin resistance; B) K. pneumoniae third-generation cephalosporin resistance; C) K. pneumoniae multidrug resistance;

D) Escherichia coli ampicillin-gentamicin resistance; E) E. coli third-generation cephalosporin resistance; F) E. coli multidrug resistance;

G) Salmonella enterica serotype Typhi fluoroquinolone resistance; H) Staphylococcus aureus methicillin resistance; I) Streptococcus pneumoniae penicillin resistance. Isolates were defined as hospital-acquired if taken $>48$ hours after patient admission. Error bars indicate $95 \%$ Cls.

this population have been reported elsewhere (CE Moore, CM Parry, P Turner, NPJ Day, N Stoesser, unpub data; N Stoesser, C Turner, P Turner, BS Cooper, unpub data), whereas whole-genome sequencing of invasive isolates is ongoing. Loss of antimicrobial selective pressure leading to declining resistance may result from changes in national/ regional antimicrobial supply or lack of active drug in antimicrobials used (19).

The number of hospital-acquired $K$. pneumoniae isolates peaked during 2011-2012. This peak may be the result of a genuine rise in the rate of hospital-acquired infections or the increased rate of blood culture sampling compared with previous years. From 2011-2012 onward, the proportion of resistant hospital-acquired K. pneumoniae isolates declined. This drop may be linked to maturation of a hospitalwide infection-control program implemented in 2010 (20) and enforced by prospective hospital-acquired infection surveillance from 2015 onward (21) or to the clinical microbiology service operating since 2012 with a strong focus on antimicrobial drug stewardship. Indeed, a recent study of prescribing practices at this hospital found $84 \%-89 \%$ of antimicrobial drug prescriptions were appropriate (22). The apparent success of these interventions suggests that they could be useful for combating AMR in similar settings. The perceived temporal drop in K. pneumoniae resistance could also be attributable to changing proportions of isolates from neonates over time; $46 \%-50 \%$ of isolates were from neonates in 2011-2014, dropping to 35\% in 2015-2016. 

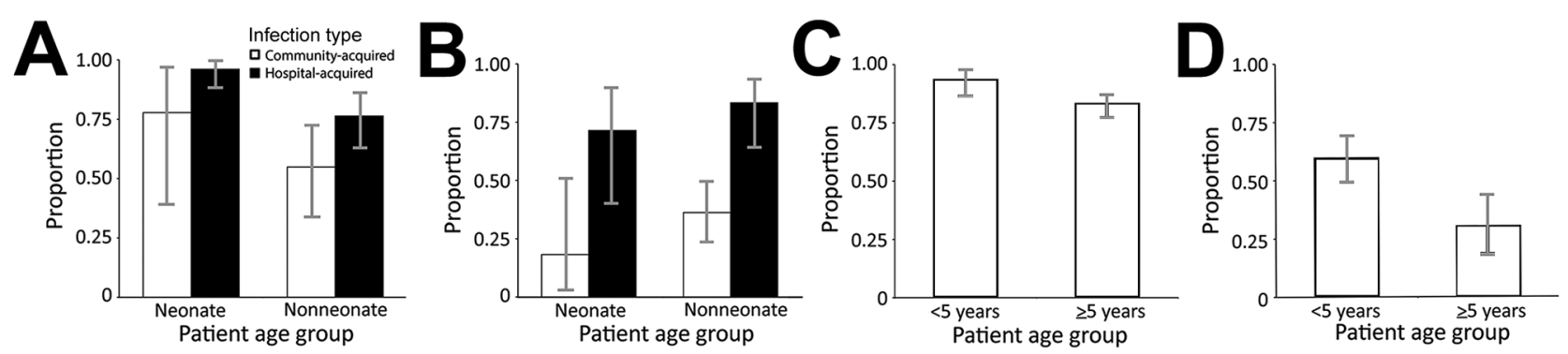

Figure 2. Antimicrobial resistance age trends, shown as proportion of resistant isolates from community-acquired and hospitalacquired infections, by patient age group, in children at Angkor Hospital for Children, Siem Reap, Cambodia, 2007-2016. A) Klebsiella pneumoniae third-generation cephalosporin resistance; B) Escherichia coli third-generation cephalosporin resistance; C) Salmonella enterica serotype Typhi multidrug resistance; D) Streptococcus pneumoniae penicillin resistance. Ages have been grouped into neonate $(0-28 \mathrm{~d})$ versus nonneonate $(\geq 29 \mathrm{~d})$ or $<5$ years versus $\geq 5 \mathrm{y}$, as appropriate for the organism. Isolates were defined as hospital-acquired if taken $>48$ hours after admission. Error bars indicate $95 \% \mathrm{Cls}$.

This study is unusual in that it directly compares different age groups of children, revealing AMR trends associated with age. Of note, the dominant pathogen in neonates, $K$. pneumoniae, was also more often resistant in neonates. For hospital-acquired isolates, this resistance may result from horizontal acquisition of resistant gramnegative organisms from hospital surfaces, as suggested by a recent multicenter study of sepsis in neonates (2). Indeed, colonization of neonates by resistant gram-negative organisms has been shown to be common at Angkor Hospital for Children and associated with subsequent invasive infection (23). For community-acquired isolates, vertical maternal transfer of resistant organisms may have a substantial role and is currently under investigation at this center.

Similarly, Salmonella Typhi from children $<5$ years of age was more often multidrug resistant than that from those $\geq 5$ years of age. Isolates from younger children have greater genetic diversity (24), although how this diversity relates to increased AMR requires further investigation. In Cambodia, the most common indication for antimicrobial drug use is infections in children $<5$ years of age; thus, children in this age group may be exposed to more antimicrobial drugs, leading to greater resistance in organisms causing infection. That $S$. pneumoniae isolates were more often penicillin resistant in children $<5$ years of age is consistent with findings of previous work showing greater colonization of this age group by multidrug-resistant pneumococci (25). Vaccination may have a collateral benefit of reducing AMR (26), which suggests that it could be useful for combating Salmonella Typhi and S. pneumoniae resistance in low- and middle-income countries. Because $85 \%$ of Salmonella Typhi isolates are simultaneously fluoroquinolone resistant and multidrug resistant, few agents remain for treating typhoid in this population, placing even greater value on preventive measures such as vaccination. In January 2015 , a 13 -valent pneumococcal conjugate vaccine was introduced in Cambodia (27) with no catch-up campaign, meaning that only $S$. pneumoniae isolates from children born in or after December 2014 could have been affected, equating to 5 isolates in this dataset. Pneumococcal vaccination is thus unlikely to have had an appreciable effect on the AMR trends reported here.

The WHO Global Report on Surveillance identified a major gap in research comparing resource use in resistant versus nonresistant pathogens (28), an area that we addressed by demonstrating that resistance is associated with worse healthcare outcomes, including increased deaths and ICU admissions, delayed effective treatment, and more than doubled admission costs. Use of patient records allows these estimates to more closely reflect reality than modeled or ecologic analyses, although it is unclear whether this increased risk for adverse outcomes represents greater virulence, delayed treatment, or confounding. The observed outcome differences between Enterobacteriaceae and A. baumannii infections suggest either a true difference in virulence or that a proportion of $A$. baumannii isolates were contaminants, an uncertainty that highlights the difficulty of establishing the clinical significance of skin-colonizing organisms.

This study has several limitations. The data derive from a single nongovernmental hospital for children with limited numbers of isolates for some bacterial species; thus, trends and outcomes may not be representative of the wider region. The study was retrospective, and classification of community-acquired and hospital-acquired infections was limited by hospital database and clinical case note accuracy, meaning that some community-acquired infections may have actually been hospital-acquired infections. Widespread prehospitalization use of antimicrobials may have selected for resistant organisms (29). There were no restrictions to blood culture submission over time, but from early 2016 onward, clinicians were asked to focus on children requiring admission, which may have affected certain 
Table 6. Multivariable logistic regression analysis of 129 hospital admission episodes for community-acquired monomicrobial gramnegative bacteremia in children at Angkor Hospital for Children, Siem Reap, Cambodia, 2007-2016*

\begin{tabular}{|c|c|c|c|c|}
\hline \multirow[b]{2}{*}{ Predictor variable } & \multicolumn{2}{|c|}{ Death } & \multicolumn{2}{|c|}{ ICU admission } \\
\hline & OR $(95 \% \mathrm{Cl})$ & $p$ value & OR $(95 \% \mathrm{Cl})$ & $\mathrm{p}$ value \\
\hline Third-generation cephalosporin resistance & $2.65(1.05-6.96)$ & 0.042 & $3.17(1.31-8.10)$ & 0.013 \\
\hline Neonate† & $3.03(1.14-8.31)$ & 0.028 & $4.56(1.83-12.16)$ & 0.002 \\
\hline Male & $0.81(0.32-2.07)$ & 0.659 & $0.81(0.35-1.85)$ & 0.616 \\
\hline Enterobacteriaceaeł & $26.25(4.43-511.1)$ & 0.003 & 3.07 (1.05-9.67) & 0.046 \\
\hline Malnourished§ & $2.11(0.85-5.35)$ & 0.111 & $2.19(0.98-5.01)$ & 0.059 \\
\hline Age $<10 y$ & $2.76(0.40-56.29)$ & 0.377 & $2.80(0.60-20.70)$ & 0.235 \\
\hline $\begin{array}{l}\text { *Analysis used outcome (death or recovery) an } \\
+0-28 \text { d of age. } \\
\ddagger \text { Acinetobacter baumannii } n=29 ; \text { Enterobacte } \\
\text { Enterobacteriaceae [consisting of Citrobacter, } \\
\S \text { Children }<10 \text { y of age only. }\end{array}$ & $\begin{array}{l}\mathrm{mission} \text { as the dep } \\
\mathrm{n}=100 \text { (consisting } \\
\text { cter, Escherichia, } K\end{array}$ & $I_{c}$ & $\begin{array}{l}\text { are unit; OR, odds } \\
\text { siella pneumoniae } \\
\text { oteus, and Serratic }\end{array}$ & er patt \\
\hline
\end{tabular}

organisms (e.g., Salmonella Typhi). Microbiology practice variations over time meant that antimicrobial susceptibility testing was not consistent; however, we believe that the value of examining the evidence over a long period outweighed the effect that these variations may have had on results. For example, in 2009, the Clinical and Laboratory Standards Institute sensitivity zone size cutoffs for carbapenems and cephalosporins in Enterobacteriaceae increased, which could have resulted in a small number of isolates previously classed as sensitive being reclassified as resistant. The reported pre-2009 resistance levels are thus conservative and would not negate the downward resistance trends observed. In the outcome analysis, we did not consider prehospitalization factors, clinical diagnosis, and non-HIV/malnutrition co-occurring conditions because quantifying those could have introduced substantial reporting bias. Furthermore, our cost estimates may be higher than actual costs because we did not account for partial/ shared doses and price fluctuations, suggesting that these cost estimates are most useful as a relative indication of cost burden.

In conclusion, the high rate of AMR in this setting of hospitalized children in Cambodia was associated with increased deaths and healthcare costs and threatens the effectiveness of first-line sepsis treatment. AMR represents a major threat to children's health globally $(5,6)$, yet there is a dearth of data for children in low-resource settings (3-6). By reporting a decade of continuous AMR surveillance data, this study fills a gap in the understanding of antimicrobial drug resistance in children in Cambodia. In the context of the current global drive to combat AMR and the goal of the Fleming Fund to improve surveillance in low- and middle-income countries, our study demonstrates the feasibility and utility of undertaking accurate long-term antimicrobial drug resistance surveillance in these countries. The methods used here are reproducible in similar low-resource settings.

\section{Acknowledgments}

We thank the clinical, laboratory, and support staff at Angkor Hospital for Children.
The Cambodia-Oxford Medical Research Unit/Angkor Hospital for Children microbiology laboratory is funded by grants from the University of Oxford-Li Ka Shing Foundation Global Health Programme, the Kadoorie Charitable Foundation, and the Wellcome Trust as part of the Wellcome Trust-Mahidol University-Oxford Tropical Medicine Research Programme.

\section{About the Author}

Dr. Fox-Lewis is a research clinician at the CambodiaOxford Medical Research Unit, Angkor Hospital for Children, Siem Reap, Cambodia. His primary research interest is antimicrobial resistance.

\section{References}

1. Liu L, Oza S, Hogan D, Chu Y, Perin J, Zhu J, et al. Global, regional, and national causes of under-5 mortality in 2000-15: an updated systematic analysis with implications for the Sustainable Development Goals. Lancet. 2016;388:3027-35. http://dx.doi.org/10.1016/S0140-6736(16)31593-8

2. Investigators of the Delhi Neonatal Infection Study (DeNIS) collaboration. Characterisation and antimicrobial resistance of sepsis pathogens in neonates born in tertiary care centres in Delhi, India: a cohort study. Lancet Glob Health. 2016;4:e752-60. http://dx.doi.org/10.1016/S2214-109X(16)30148-6

3. Lubell Y, Ashley EA, Turner C, Turner P, White NJ. Susceptibility of community-acquired pathogens to antibiotics in Africa and Asia in neonates - an alarmingly short review. Trop Med Int Health. 2011;16:145-51. http://dx.doi.org/10.1111/ j.1365-3156.2010.02686.x

4. Downie L, Armiento R, Subhi R, Kelly J, Clifford V, Duke T. Community-acquired neonatal and infant sepsis in developing countries: efficacy of WHO's currently recommended antibioticssystematic review and meta-analysis. Arch Dis Child. 2013;98: 146-54. http://dx.doi.org/10.1136/archdischild-2012-302033

5. Huynh BT, Padget M, Garin B, Herindrainy P, Kermorvant-Duchemin E, Watier L, et al. Burden of bacterial resistance among neonatal infections in low income countries: how convincing is the epidemiological evidence? BMC Infect Dis. 2015;15:127. http://dx.doi.org/10.1186/s12879-015-0843-x

6. Le Doare K, Bielicki J, Heath PT, Sharland M. Systematic review of antibiotic resistance rates among gram-negative bacteria in children with sepsis in resource-limited countries. J Pediatric Infect Dis Soc. 2015;4:11-20. http://dx.doi.org/10.1093/jpids/piu014

7. O'Neill J. Tackling drug-resistant infections globally: final report and recommendations [cited 2017 Jul 24]. https://amr-review.org/ Publications.html 
8. Department of Health and Social Care UK. Tackling antibiotics resistance in low income countries [cited $2017 \mathrm{Jul} 24]$. https://www.gov.uk/government/news/tackling-antibioticsresistance-in-low-income-countries

9. World Health Organization. Global antimicrobial resistance surveillance system: manual for early implementation [cited 2017 Jul 24]. http://www.who.int/antimicrobial-resistance/publications/ surveillance-system-manual/en/

10. Lim C, Takahashi E, Hongsuwan M, Wuthiekanun V, Thamlikitkul V, Hinjoy S, et al. Epidemiology and burden of multidrug-resistant bacterial infection in a developing country. eLife. 2016;5:5. http://dx.doi.org/10.7554/eLife.18082

11. Chheng K, Carter MJ, Emary K, Chanpheaktra N, Moore CE, Stoesser N, et al. A prospective study of the causes of febrile illness requiring hospitalization in children in Cambodia. PLoS One. 2013;8:e60634. http://dx.doi.org/10.1371/ journal.pone.0060634

12. Asian Development Bank. Cambodia country poverty analysis [cited 2017 Jul 24]. https://www.adb.org/sites/default/files/ institutional-document/151706/cambodia-country-povertyanalysis-2014.pdf

13. Stoesser N, Moore CE, Pocock JM, An KP, Emary K, Carter M, et al. Pediatric bloodstream infections in Cambodia, 2007 to 2011. Pediatr Infect Dis J. 2013;32:e272-6. http://dx.doi.org/10.1097/ INF.0b013e31828ba7c6

14. Clinical and Laboratory Standards Institute. Performance standards for antimicrobial disk susceptibility tests, 27th edition (M100S27). Wayne (PA): The Institute; 2017.

15. The R Project for Statistical Computing. Vienna, Austria [cited 2017 Jul 24]. https://www.r-project.org/

16. Ashley EA, Lubell Y, White NJ, Turner P. Antimicrobial susceptibility of bacterial isolates from community acquired infections in sub-Saharan Africa and Asian low and middle income countries. Trop Med Int Health. 2011;16:1167-79. http://dx.doi.org/ 10.1111/j.1365-3156.2011.02822.x

17. Kim SH, Song JH, Chung DR, Thamlikitkul V, Yang Y, Wang H, et al.; ANSORP Study Group. Changing trends in antimicrobial resistance and serotypes of Streptococcus pneumoniae isolates in Asian countries: an Asian Network for Surveillance of Resistant Pathogens (ANSORP) study. Antimicrob Agents Chemother. 2012;56:1418-26. http://dx.doi.org/10.1128/AAC.05658-11

18. Song JH, Hsueh PR, Chung DR, Ko KS, Kang CI, Peck KR, et al.; ANSORP Study Group. Spread of methicillin-resistant Staphylococcus aureus between the community and the hospitals in Asian countries: an ANSORP study. J Antimicrob Chemother. 2011;66:1061-9. http://dx.doi.org/ $10.1093 / \mathrm{jac} / \mathrm{dkr} 024$

19. Newton PN, Green MD, Fernández FM, Day NP, White NJ. Counterfeit anti-infective drugs. Lancet Infect Dis. 2006;6:602-13. http://dx.doi.org/10.1016/S1473-3099(06)70581-3
20. Stoesser N, Emary K, Soklin S, Peng An K, Sophal S, Chhomrath $\mathrm{S}$, et al. The value of intermittent point-prevalence surveys of healthcare-associated infections for evaluating infection control interventions at Angkor Hospital for Children, Siem Reap, Cambodia. Trans R Soc Trop Med Hyg. 2013;107:248-53. http://dx.doi.org/10.1093/trstmh/trt005

21. Hearn P, Miliya T, Seng S, Ngoun C, Day NPJ, Lubell Y, et al. Prospective surveillance of healthcare associated infections in a Cambodian pediatric hospital. Antimicrob Resist Infect Control. 2017;6:16. http://dx.doi.org/10.1186/s13756-017-0172-5

22. Fox-Lewis S, Pol S, Miliya T, Day NPJ, Turner P, Turner C. Utilization of a clinical microbiology service at a Cambodian paediatric hospital and its impact on appropriate antimicrobial prescribing. J Antimicrob Chemother. 2017.

23. Turner P, Pol S, Soeng S, Sar P, Neou L, Chea P, et al. high prevalence of antimicrobial-resistant gram-negative colonization in hospitalized Cambodian infants. Pediatr Infect Dis J. 2016;35:856-61. http://dx.doi.org/10.1097/ INF.0000000000001187

24. Holt KE, Baker S, Dongol S, Basnyat B, Adhikari N, Thorson S, et al. High-throughput bacterial SNP typing identifies distinct clusters of Salmonella Typhi causing typhoid in Nepalese children. BMC Infect Dis. 2010;10:144. http://dx.doi.org/10.1186/ 1471-2334-10-144

25. Turner P, Turner C, Suy K, Soeng S, Ly S, Miliya T, et al. Pneumococcal infection among children before introduction of 13-valent pneumococcal conjugate vaccine, Cambodia. Emerg Infect Dis. 2015;21:2080-3. http://dx.doi.org/10.3201/ eid2111.150914

26. Dagan R, Klugman KP. Impact of conjugate pneumococcal vaccines on antibiotic resistance. Lancet Infect Dis. 2008;8:785-95. http://dx.doi.org/10.1016/S1473-3099(08)70281-0

27. Moore CE, Giess A, Soeng S, Sar P, Kumar V, Nhoung P, et al. Characterisation of invasive Streptococcus pneumoniae isolated from Cambodian children between 2007-2012. PLoS One. 2016;11:e159358. http://dx.doi.org/10.1371/journal.pone.0159358

28. World Health Organization. Antimicrobial resistance: global report on surveillance 2014 [cited $2017 \mathrm{Jul}$. http://www.who.int/ drugresistance/documents/surveillancereport/en/

29. Om C, Daily F, Vlieghe E, McLaughlin JC, McLaws ML. Pervasive antibiotic misuse in the Cambodian community: antibiotic-seeking behaviour with unrestricted access. Antimicrob Resist Infect Control. 2017;6:30. http://dx.doi.org/10.1186/ s13756-017-0187-y

Address for correspondence Andrew Fox-Lewis, Cambodia-Oxford Medical Research Unit, Angkor Hospital for Children, Tep Vong (Achamean) Rd and Oum Chhay St, Svay Dangkum, PO Box 50, Siem Reap, Cambodia; email: afoxlewis@gmail.com 